\title{
Primary Percutaneous Coronary Intervention Using BaLloon- Assisted Sliding and Tracking (BLAST) of Diagnostic Catheter to Negotiate Double Hairpin Loop of Radial Artery
}

\author{
Santosh Kumar Sinha ${ }^{a}$,, Vikas Mishra ${ }^{a}$, Mukesh Jitendra Jha ${ }^{a}$, Mahmadula Razia , Anupam Mahrotra ${ }^{a}$, \\ Nasar Abdali ${ }^{\mathrm{a}}$, Lokendra Rekwal ${ }^{\mathrm{a}}$, Vikas Chaturvedi ${ }^{\mathrm{a}}$, Anupam Kumar Singha ${ }^{\mathrm{a}}$, Ramesh Thakur ${ }^{\mathrm{a}}$
}

\begin{abstract}
During transradial intervention, sharp edge of the guide catheter tip may act like a "razor-blade" and can prevent the catheter navigation especially in situation like double hair pin loop. Here, we report primary percutaneous coronary intervention (PCI) through diagnostic catheter using an innovative technique, balloon-assisted sliding and tracking (BLAST), to overcome this double hairpin loop, thus saving time and contrast volume.
\end{abstract}

Keywords: Balloon-assisted sliding and tracking; Double hair pin loop; Razor-effect; Primary percutaneous coronary intervention

\section{Introduction}

Following the first reports of radial coronary angiography by Lucian Campeau in 1989 and radial percutaneous coronary intervention (PCI) by Ferdinand Kiemeneij in 1992, there has been an increase in the use of transradial catheterization (TRC) around the world with reduced access site bleeding complications, mortality and healthcare costs $[1,2]$. The presence of a radial artery loop is the most common cause of transradial failure with incidence of a full $360^{\circ}$ radial artery loop around $2-4 \%$ in different series [3-5]. Therefore, it is considered as "Achilles' heel" of this technique. In these situations, the sharp edge of the guide catheter tip produces a "razor-blade effect" which can prevent the guide catheter navigation and sometimes can lead to the radial artery spasm, perforation and forearm hematoma formation [6]. Various techniques such as balloon-assisted tracking (BAT) have been described to overcome such situations $[7,8]$. We describe an innovative technique to

Manuscript accepted for publication March 21, 2017

a Department of Cardiology, LPS Institute of Cardiology, G.S.V.M. Medical College, Kanpur, Uttar Pradesh 208002, India

${ }^{b}$ Corresponding Author: Santosh Kumar Sinha, Department of Cardiology, LPS Institute of Cardiology, G.S.V.M. Medical College, Kanpur, Uttar Pradesh 208002, India. Email: fionasan@rediffmail.com

doi: https://doi.org/10.14740/cr540w circumvent such difficulty where even BAT fails.

\section{Case Report}

A 41-year-old male with past history of hypertension and dyslipidemia presented with retrosternal chest discomfort and sweating of $2 \mathrm{~h}$ duration. His physical examinations and biochemistry were all unremarkable. Electrocardiogram revealed ST elevation in II, III, and aVF with reciprocal changes in I and aVL. Echocardiography revealed mild hypokinesia in left circumflex territory with ejection fraction of $47 \%$. He was taken for primary PCI after proper consent. He was preloaded with prasugrel $60 \mathrm{mg}$, aspirin $325 \mathrm{mg}$ and atorvastatin $80 \mathrm{mg}$. Right radial artery was punctured by $21 \mathrm{G}$ needle, 0.021" guidewires (Avanti transradial kit; Cordis Corp., USA) were inserted and 6-F sheath was placed. After sheath replacement, cocktail containing $200 \mu \mathrm{g}$ nitroglycerin, 2.5 $\mathrm{mg}$ diltiazem, and 2,500 IU unfractioned heparin was injected. While pushing diagnostic catheter, great difficulty was encountered as it was not going forward. Check angiogram revealed double hairpin turn with $360^{\circ}$ loop (Fig. 1). First, we tried to negotiate the turn by using $0.035^{\prime \prime}$ hydrophilic wire (Terumo, Japan) but failed. A 0.014" PTCA guidewire (balance middle weight (BMW)) was used to negotiate the loop which nearly crossed the loop (Fig. 2) and advanced into brachial artery (Fig. 3). Diagnostic catheter was advanced over the wire which negotiated the first hairpin turn of the loop, but refused to budge any further. Another 0.014 " PTCA guidewire (buddy wire) was used by advancing into distal part of brachial artery but could not negotiate the loop. We tried balloon-assisted technique but because of razor effect and $360^{\circ}$ loop, it failed (Fig. 4a). We then pushed $1.5 \times 10$ $\mathrm{mm}$ balloon (Sapphire, OrbusNeich, the Netherlands) little further from catheter tip and inflated up to 3 atm pressure by keeping the distal end of the wire into brachial artery. Holding the balloon-wire assembly firm, we advanced the catheter by gently sliding over the inflated balloon, thus negotiating the first turn (Supplementary video 1, www.cardiologyres. org). We deflated the balloon and again pushed little ahead of the catheter. Once again, we inflated the balloon at $3 \mathrm{~atm}$ pressure and catheter was advanced by gently sliding over the inflated balloon, thus successfully negotiating the $360^{\circ}$ loop overcoming razor effect of tip of diagnostic catheter 


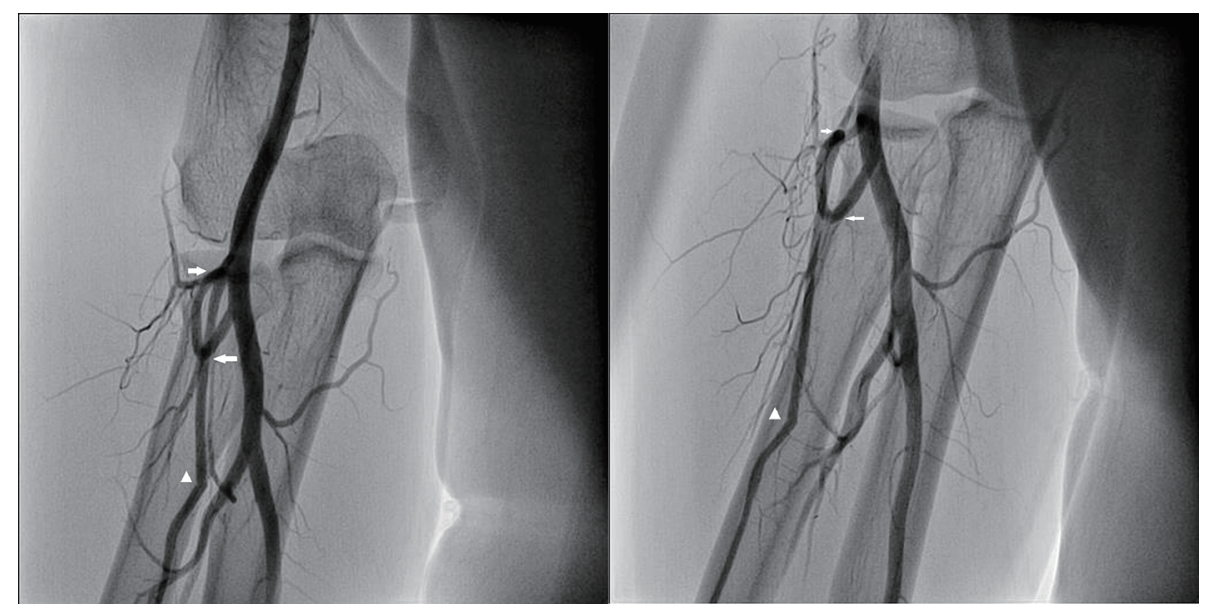

Figure 1. Double hairpin turn (white arrow) with $360^{\circ}$ loop of radial artery (arrow head showing tip of the diagnostic catheter).

(Supplementary video 2, www.cardiologyres.org, Fig. 4b). Once crossed the loop, we gently pulled back the entire assembly which straightened the loop and eased the further advancement of the catheter. We pulled out the balloon and the procedure was completed in usual fashion. Coronary angiogram revealed normal left main, left anterior descending artery, right coronary artery arising from left sinus and subtotal occlusion of proximal circumflex artery (Fig. 5a, b). PCI was planned of the culprit artery and 7,000 $\mathrm{U}$ of heparin was given further. Since great difficulty was encountered while advancing the diagnostic catheter, we planned intervention through the same diagnostic catheter to cut down the time, to avoid vasospasm while multiple catheter exchange and contrast overload. BMW guidewires 0.014", 190 cm (Abott, USA) were parked beyond the lesion and were pre-dilated with $2 \times 10 \mathrm{~mm}$ Minitrak balloon (Abott, USA) to $12 \mathrm{~atm}$ (Fig. 5c). It was stented by deploying $2.75 \times 21 \mathrm{~mm}$ Xience

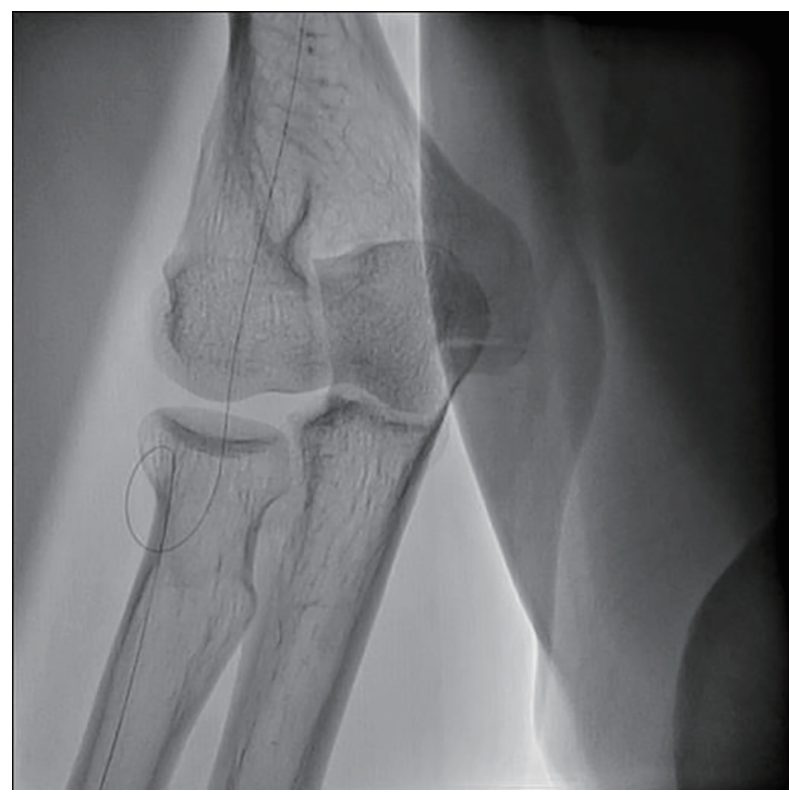

Figure 2. BMW guidewire (0.014") after crossing the complex loop.
Prime stent (everolimus drug-eluting stent, Abott, USA) up to $13 \mathrm{~atm}$ pressure (Fig. 5d) and further post-dilated by 2.75 $\times 10 \mathrm{~mm}$ Minitrak non-compliant balloon up to 20 atm pressure achieving TIMI 3 flow (Figs. 6a, b, c, d, and 7). His symptoms and ECG stabilized. Contrast angiography of the involved segment of the vasculature was performed to rule out any perforation after removal of catheter. Sheath was removed and compression was performed for $2 \mathrm{~h}$ with a radial compression device (TR band; Terumo, Inc.) using the "patent haemostasis" protocol. TR band was removed after $2 \mathrm{~h}$ of sheath removal and a light pressure bandage was applied which was removed next day. He was discharged on the third day with aspirin $150 \mathrm{mg} /$ day, prasugrel $10 \mathrm{mg}$ /day, atorvastatin $80 \mathrm{mg} /$ day, metoprolol $100 \mathrm{mg}$ /day and ramipril $2.5 \mathrm{mg} /$ day. Patient is doing fine since then with regular follow-up at our institute.

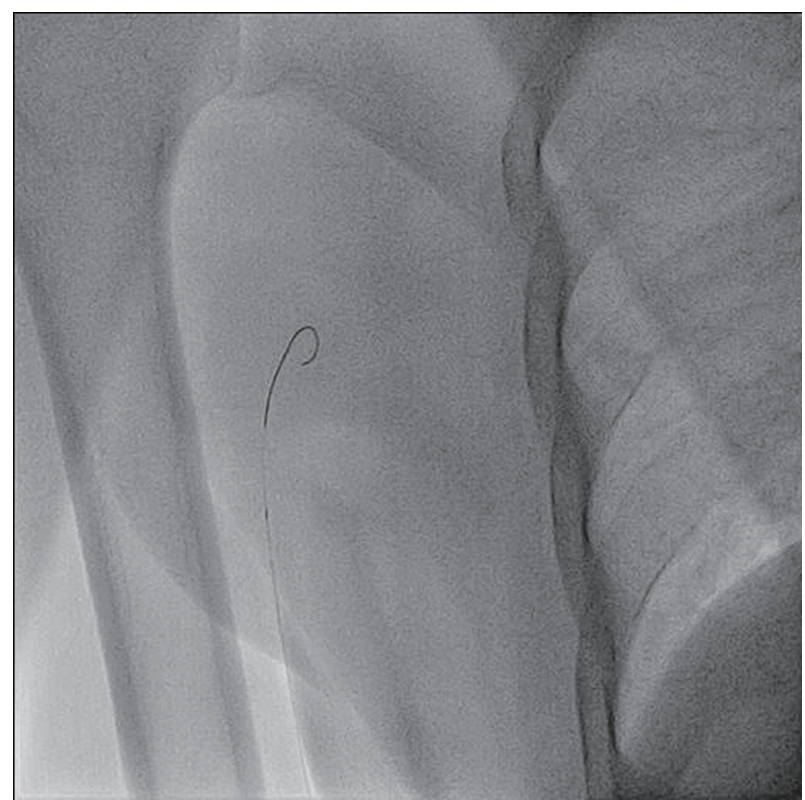

Figure 3. BMW guidewire (0.014") after crossing the complex loop and parked distally into brachial artery. 


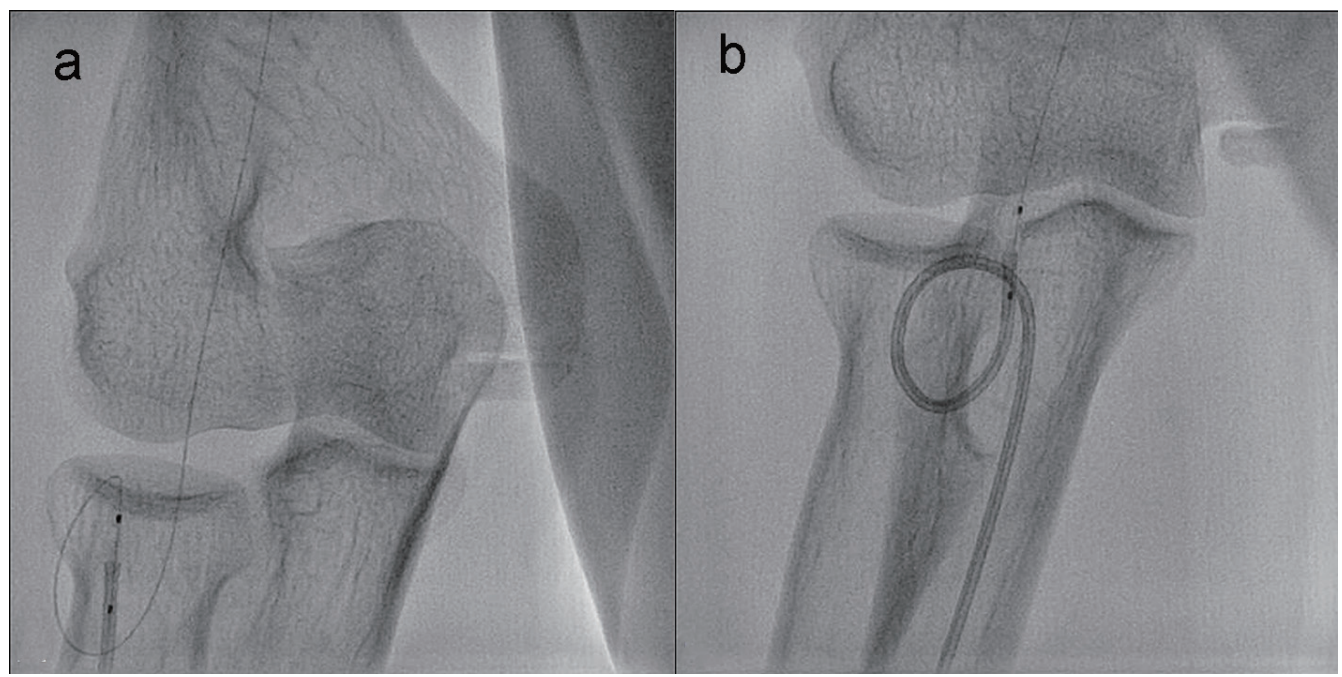

Figure 4. Catheter failing cross the first turn because of razor effect despite use of balloon-assisted tracking (a). Catheter successfully negotiated the $360^{\circ}$ loop overcoming razor effect of tip of catheter after BLAST (b).

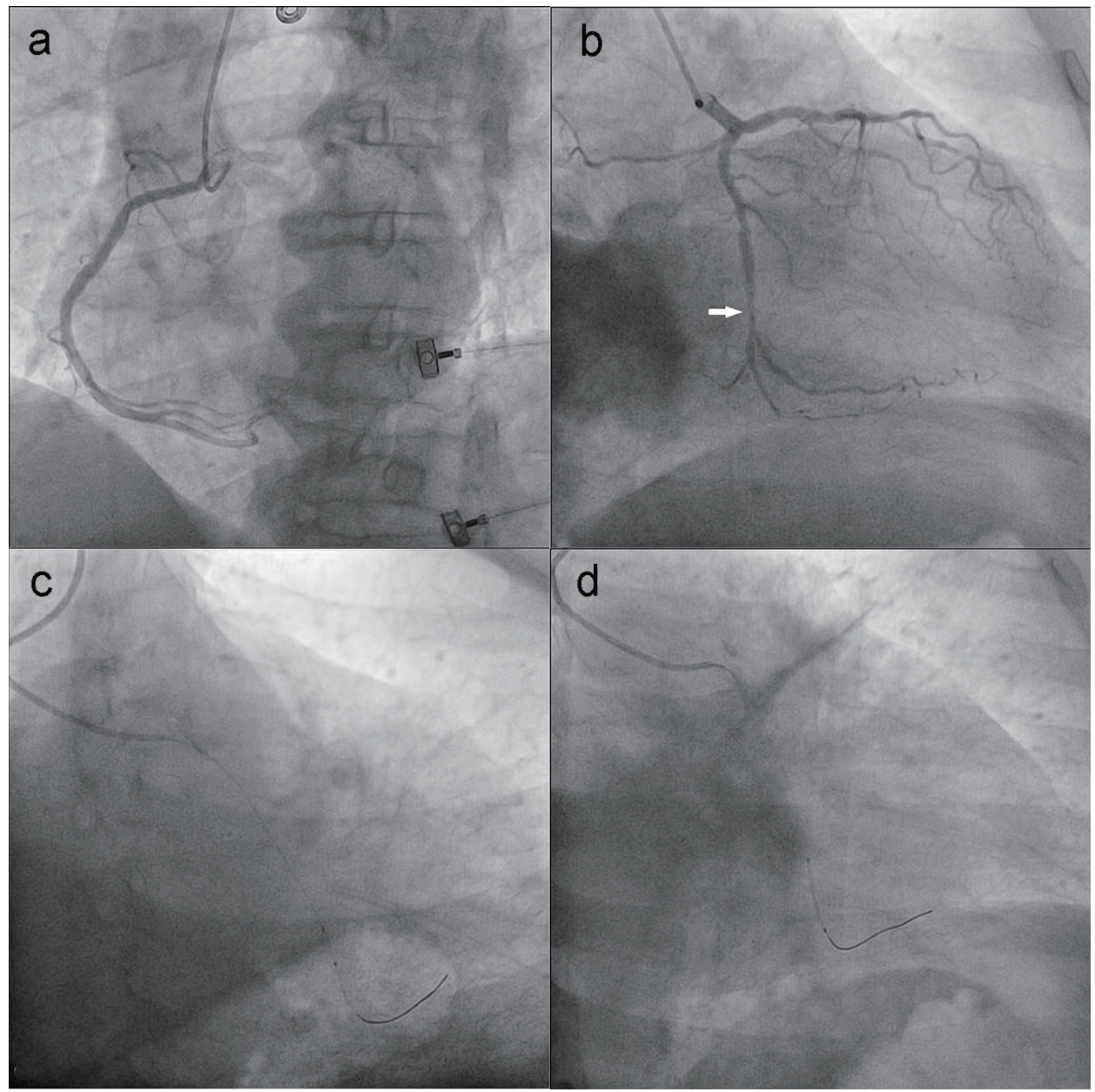

Figure 5. Coronary angiogram revealed normal left main, left anterior descending artery, right coronary artery arising from left sinus and subtotal occlusion of proximal circumflex artery (a, b). Pre-dilatation of lesion by semi compliant balloon (c). Stent ( 2.75 $\times 21 \mathrm{~mm}$ Xience prime) being deployed (d). 


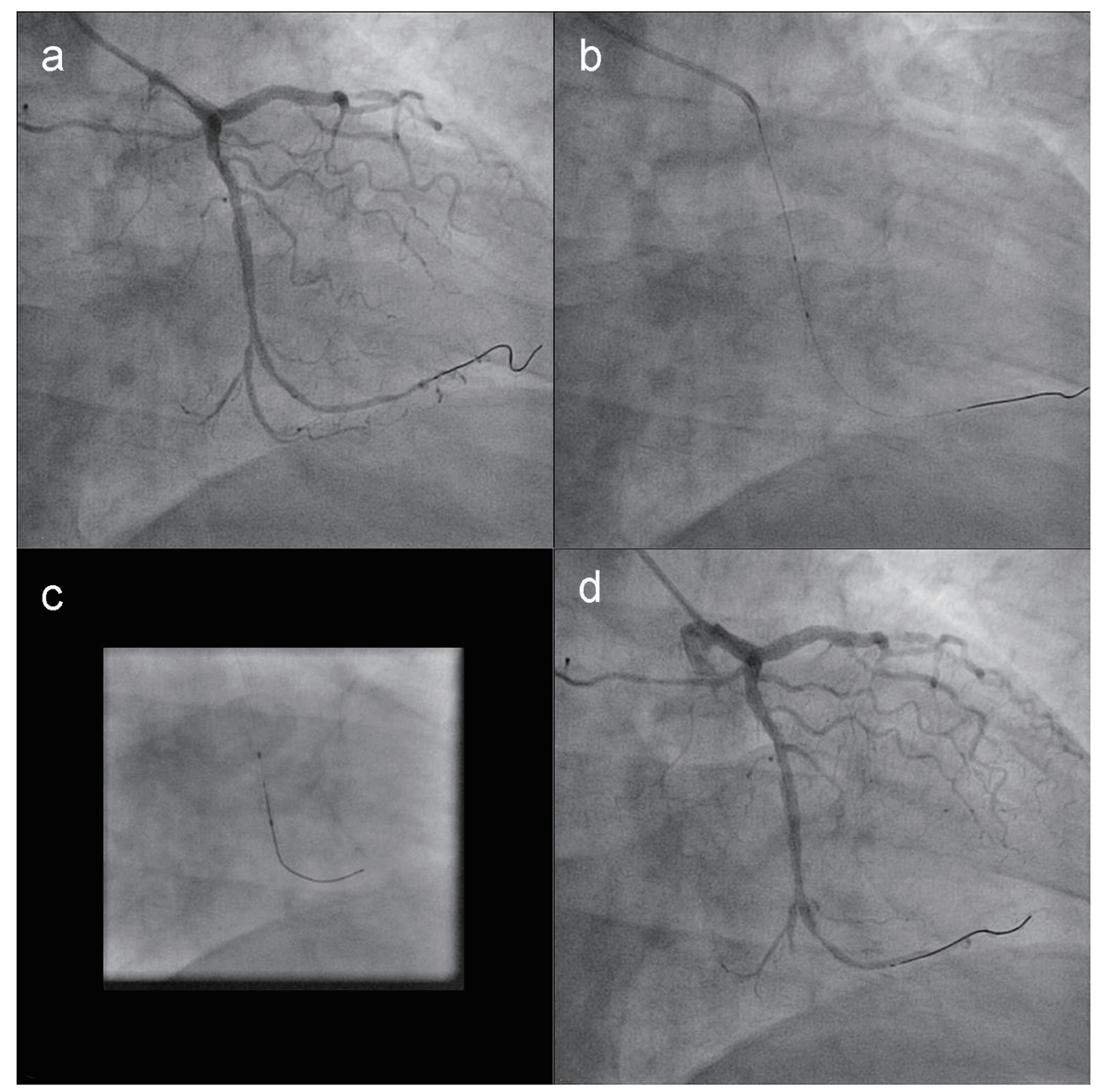

Figure 6. Post-stenting circumflex artery (a). Post-dilatation of stent by non-compliant balloon (b, c). Post-stenting circumflex artery (d).

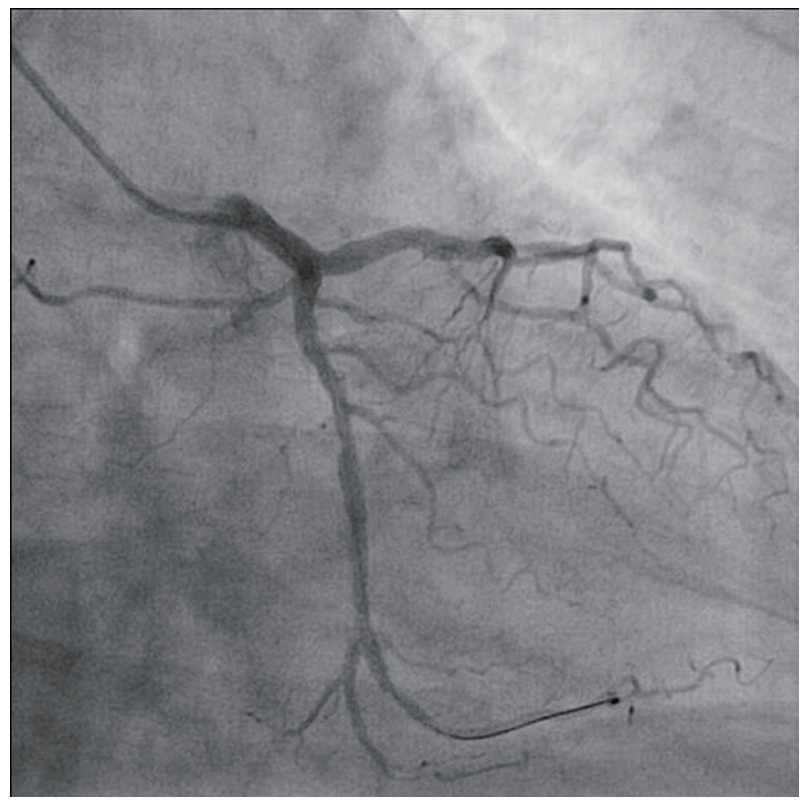

Figure 7. Final TIMI 3 of culprit artery.

\section{Discussion}

Difficult vascular anatomy is the most important cause of technical failure during TRC. Catheter manipulation becomes difficult and painful even after successful crossing of the affected segment creating a vicious cycle of pain and vasospasm leading to further difficulty. Therefore, double hairpin turn creating $360^{\circ}$ loop is considered as the "Achilles' heel" of TRC.

Various techniques have been described to circumvent these situations [4, 7-9]. Despite the application of these techniques, some anatomical situations cannot be overcome. As reported by Patel et al [7], there were three technical failures by BAT technique out of more than 8,000 TRA procedures. Conical shape, tapered tip, and smooth surface of an inflated balloon and its partially protruding segment during BAT help non-traumatic advancement of a catheter through small calibre of RA negating the "razor effect" of the distal catheter tip and related damage to vasculature. Also, it increases flexibility of assembly and helps the catheter to traverse through complex curves. The potential disadvantage with BAT is that it increases the total length of the assembly (catheter tip-partially protruding balloon) that needs to cruise through the double 
loop; therefore it may not be win-win situation all the time. In BLAST, partially inflated balloon at low pressure (3 atm) smoothens the pushability and trackibility as total length of the catheter needs to cruise through the loop does not change. It therefore forms small arc of a small circle rather big arc as noted in BAT. The low pressure balloon inflation keeps balloon tip more flexible hence, it can take the complex curves and overcome obstructions with much ease. Compared to BAT, we advise that smaller semi compliant balloon $(6-8 \mathrm{~mm})$ which may be used in BLAST to facilitate catheter movement. Use of 5-F diagnostic catheter as a guiding catheter for PCI as first reported by Salinger et al [10] is becoming increasingly popular due to the increasing trends toward slender catheter for transradial approach. It has advantage of smaller arterial punctures and thus decreases morbidity, allows early mobilization and discharge, less contrast volume and therefore reduces potential nephrotoxicity. As time is most vital component of PCI to achieve shortest possible door to balloon time, presence of complex radial loop and culprit lesion was discrete, PCI was performed by diagnostic catheter only. It has also been noted that guiding catheters used for PCI may sometimes not be able to selectively engage the coronary ostium even though an earlier successful cannulation with its diagnostic counterpart could be obtained because of the slight differences in shape between the two catheters in form of shorter tip and lack of tip tapering for the guiding catheter, which may result in multiple guide selection attempts [11]. Potential drawback with the use of 5-F catheters has been unsatisfactory vessel opacification, need for deep engagement, which may be associated with coronary dissections, and poor support complex interventions; type A lesion can easily be treated by 5-F diagnostic catheter. We had adequate vessel opacification at all times enabling us to accurately position and implant the stent without increasing the total radiation time or dye consumption. Sufficient support was achieved without the need for deep catheter intubation with easy positioning of the stent across the stenotic lesion. In conclusion, PCI via a 5-F diagnostic catheter is technically feasible, and allows for significant resource savings, taking into consideration the complex radial loop with $360^{\circ}$ turn. It is an attractive technical alternate in selected cases.

\section{Conflicts of Interest}

None.

\section{References}

1. Kiemeneij F, Laarman GJ, de Melker E. Transradial artery coronary angioplasty. Am Heart J. 1995;129(1):1-7.

2. Jolly SS, Yusuf S, Cairns J, Niemela K, Xavier D, Widimsky P, Budaj A, et al. Radial versus femoral access for coronary angiography and intervention in patients with acute coronary syndromes (RIVAL): a randomised, parallel group, multicentre trial. Lancet. 2011;377(9775):14091420 .

3. Lo TS, Nolan J, Fountzopoulos E, Behan M, Butler R, Hetherington SL, Vijayalakshmi K, et al. Radial artery anomaly and its influence on transradial coronary procedural outcome. Heart. 2009;95(5):410-415.

4. Barbeau GR. Radial loop and extreme vessel tortuosity in the transradial approach: advantage of hydrophilic-coated guidewires and catheters. Catheter Cardiovasc Interv. 2003;59(4):442-450.

5. Dehghani P, Mohammad A, Bajaj R, Hong T, Suen CM, Sharieff W, Chisholm RJ, et al. Mechanism and predictors of failed transradial approach for percutaneous coronary interventions. JACC Cardiovasc Interv. 2009;2(11):10571064.

6. Patel T, Shah S, Sanghavi K, Pancholy S. Management of radial and brachial artery perforations during transradial procedures - a practical approach. J Invasive Cardiol. 2009;21(10):544-547.

7. Patel T, Shah S, Pancholy S, Rao S, Bertrand OF, Kwan T. Balloon-assisted tracking: a must-know technique to overcome difficult anatomy during transradial approach. Catheter Cardiovasc Interv. 2014;83(2):211-220.

8. Patel T, Shah S, Pancholy S. Balloon-assisted tracking of a guide catheter through difficult radial anatomy: a technical report. Catheter Cardiovasc Interv. 2013;81(5):E215218.

9. Bazemore E, Mann JT, 3rd. Problems and complications of the transradial approach for coronary interventions: a review. J Invasive Cardiol. 2005;17(3):156-159.

10. Salinger MH, Kern MJ. First use of a 5 French diagnostic catheter as a guiding catheter for percutaneous transluminal coronary angioplasty. Cathet Cardiovasc Diagn. 1989;18(4):276-278.

11. Khattab AA, Shrestha NR, Meier B. Double-vessel coronary stenting via 5 French diagnostic catheters. Catheter Cardiovasc Interv. 2012;80(4):630-633. 